\title{
BMJ Open Effects of non-pharmacological and non- surgical interventions on health outcomes in systemic sclerosis: protocol for a living systematic review
}

\author{
Marie-Nicole Discepola, ${ }^{1,2}$ Andrea Carboni-Jiménez,, ${ }^{1,3}$ Linda Kwakkenbos, ${ }^{4}$ \\ Richard S Henry, ${ }^{1,3}$ Jill Boruff, ${ }^{5}$ Ankur Krishnan, ${ }^{1}$ Carina Boström, ${ }^{6}$ \\ S Nicole Culos-Reed, ${ }^{7}$ Marie Hudson, ${ }^{8}$ David M Leader, ${ }^{9}$ Malin Mattsson,, 10 \\ Luc Mouthon, ${ }^{11,12}$ Robyn Wojeck, ${ }^{13}$ Elizabeth Yakes Jimenez, ${ }^{14}$ Maureen Sauve, ${ }^{15}$ \\ Joep Welling, ${ }^{16}$ Geneviève Guillot, ${ }^{17}$ Andrea Benedetti, ${ }^{8,18,19,20}$ \\ Brett D Thombs (D) 1,3,8,18,21,22
}

To cite: Discepola M-

$\mathrm{N}$, Carboni-Jiménez A

Kwakkenbos L, et al. Effects of non-pharmacological and non-surgical interventions on health outcomes in systemic sclerosis: protocol for a living systematic review. BMJ Open 2021;11:e047428. doi:10.1136/ bmjopen-2020-047428

- Prepublication history and additional supplemental material for this paper are available online. To view these files, please visit the journal online ().

Received 28 November 2020 Revised 31 March 2021 Accepted 09 April 2021

Check for updates

(C) Author(s) (or their employer(s)) 2021. Re-use permitted under CC BY-NC. No commercial re-use. See rights and permissions. Published by BMJ.

For numbered affiliations see end of article.

Correspondence to

Dr Brett D Thombs;

brett.thombs@mcgill.ca

\section{ABSTRACT}

Introduction Systemic sclerosis (SSc; scleroderma) is a rare, chronic, autoimmune disease with a high level of burden, a significant impact on the ability to carry out daily activities, and a considerable negative impact on healthrelated quality of life. Non-pharmacological interventions could be provided to potentially improve mental and physical health outcomes. However, the effectiveness of non-pharmacological interventions on health and wellbeing among individuals with SSc has not been well established. The proposed living systematic review aims to identify and evaluate randomised controlled trial (RCT) evidence on the effectiveness of non-pharmacological and non-surgical interventions on mental and physical health outcomes and on the delivery of such services in SSc. Methods and analysis Eligible studies will be RCTs that examine non-pharmacological and non-surgical interventions aimed at improving health outcomes among individuals with SSc or the delivery of services intended to improve healthcare or support of people with SSc (eg, support groups). All RCTs included in a previous systematic review that sought studies published between 1990 and March 2014 will be evaluated for inclusion. Additional trials will be sought from January 2014 onwards using a similar, augmented search strategy developed by a health sciences librarian. We will search the MEDLINE, Embase, CINAHL, PsycINFO, Cochrane Library and Web of Science databases and will not restrict by language. Two independent reviewers will determine the eligibility of identified RCTs and will extract data using a prespecified standardised form in DistillerSR. Meta-analyses will be considered if $\geq 2$ eligible RCTs report similar nonpharmacological interventions and comparable health outcomes. We will conduct a qualitative synthesis for interventions that cannot be synthesised via metaanalysis.

Ethics and dissemination We will post initial and ongoing results via a website, publish results periodically via peer-reviewed journal publication, and present results at patient-oriented events.

PROSPERO registration number CRD42020219914.
Strengths and limitations of this study

- Our living systematic review will be conducted using rigorous methods that comply with recommendations in the Cochrane Handbook for Systematic Reviews of Interventions and Cochrane guidance for living systematic reviews, will include a risk of bias assessment, and will be conducted with peer-reviewed searches developed by a research librarian.

- Our systematic review will only include randomised controlled trial (RCT) evidence in order to avoid sources of bias more common in non-randomised trials, particularly pre-post comparisons.

- Our systematic review team includes experts in a wide variety of disciplines and areas of knowledge relevant to non-pharmacological care and treatment in systemic sclerosis (SSc), as well as two patient collaborators with experience participating in research and providing first-hand insight into the needs of people with SSc.

- We will conduct a living systematic review; results will be continually updated as new evidence is published and will be distributed across wellestablished dissemination networks.

- Our search will seek eligible trials published from 2014 onwards to supplement RCTs identified by a previous systematic review, meaning it is possible, although unlikely, that studies published prior to 2014 may have been missed and will not be picked up by our searches.

\section{INTRODUCTION}

Systemic sclerosis (SSc; scleroderma) is a rare, chronic, autoimmune disease characterised by vasculopathy and excessive collagen production. ${ }^{1}$ Onset typically occurs at around 50 years, and approximately $80 \%$ of people with SSc are women. ${ }^{12}$ SSc can affect multiple 
organ systems, including the skin, lungs, gastrointestinal tract and heart. Common manifestations include Raynaud's phenomenon, skin thickening, dyspnoea and cough, gastro-oesophageal reflux and other gastrointestinal symptoms. ${ }^{12}$ Disease presentation is extremely heterogeneous, and the course of the disease is highly unpredictable. ${ }^{12}$ People with SSc commonly experience hand function and mobility limitations, pain, fatigue, sleep problems, pruritus, depression and body image distress from disfigurement (eg, skin tightening, pigment changes, hand contractures, telangiectasias). ${ }^{3-9}$ These symptoms often have a considerable negative impact on health-related quality of life. ${ }^{510} 11$ There is currently no cure for SSc. Non-pharmacological interventions (eg, psychological, educational and rehabilitation interventions) could potentially be used to improve quality of life. $^{12}$

The effects of non-pharmacological and non-surgical interventions on health and well-being among individuals with SSc has not been well established. A previous systematic review ${ }^{13}$ of the effectiveness of non-pharmacological interventions in SSc identified 23 eligible studies published between 1990 and March 2014, of which 9 were randomised controlled trials (RCTs). Included RCTs evaluated a multifaceted oral health intervention, multidisciplinary team care, and a variety of rehabilitation strategies. They all included small numbers of participants (range 25-53 total participants; median 35), and risk of bias was high in most trials.

Two more recent systematic reviews have evaluated exercise therapies ${ }^{14}$ and dietary interventions. ${ }^{15}$ A 2019 review ${ }^{14}$ included nine studies on the safety and effectiveness of exercise therapies published between 1990 and 2019, of which four were RCTs. The four RCTs evaluated hand exercises, orofacial exercises and muscle strengthening exercises. The small numbers of participants included in trials (range 4-48 total participants) and generally high risk of bias across trials, however, made it difficult to draw conclusions. A 2019 review $^{15}$ of dietary interventions for gastrointestinal symptoms in SSc included three studies, published between 2011 and 2017, but none were RCTs.

Since 2015, several larger, more robust RCTs on non-pharmacological interventions in SSc have been published, and others are in progress. Published RCTs include an evaluation of a personalised physical therapy programme $(\mathrm{N}=220),{ }^{16}$ an internet-based selfmanagement programme $(\mathrm{N}=247),{ }^{17}$ a home-based exercise programme $(\mathrm{N}=44)^{18}$ and a comparison of two educational interventions in the rehabilitation of microstomia $(\mathrm{N}=63),{ }^{19}$ for example. RCTs in progress with results expected in the next year include a web-based hand rehabilitation programme $(\mathrm{N}=466),{ }^{20}$ a supportgroup leader educational programme $(\mathrm{N}$ targeted $=180),{ }^{21}$ and a COVID-19 related mental health intervention ( $\mathrm{N}$ targeted $=162) .^{22}$

The proposed living systematic review aims to identify and evaluate RCT evidence on the effectiveness of non-pharmacological and non-surgical interventions on health outcomes, including quality of life outcomes, and on the delivery of services in SSc. This will be accomplished via an initial systematic review followed by updates as new evidence is identified. The review will facilitate decisions on non-pharmacological healthcare options for people with SSc and will identify gaps in knowledge, which will facilitate planning of future research.

\section{METHODS AND ANALYSIS}

The living systematic review was registered in the PROSPERO prospective register of systematic reviews, and any future changes to the study protocol will be registered as amendments. The protocol was developed based on methodological guidance from the Cochrane Handbook for Systematic Reviews of Interventions and Cochrane guidance for living systematic reviews. ${ }^{23}{ }^{24}$ The present protocol is reported according to Preferred Reporting Items for Systematic Review and Meta-Analysis Protocols. ${ }^{25}$ The systematic review will be reported in accordance with the Preferred Reporting Items for Systematic Reviews and Meta-Analyses statement. ${ }^{26}$ Initial searches were conducted on 30 November 2020, following registration and initial submission of this protocol for peer review. There is no planned end date.

\section{Study eligibility}

Eligible studies must be RCTs that examine nonpharmacological and non-surgical interventions aimed at improving health outcomes among individuals with SSc or the delivery of services intended to improve healthcare or support of people with SSc (eg, support groups). Non-randomised trials will be excluded, because of important limitations on the ability to draw conclusions about intervention effectiveness, particularly when done without a control group. This is because many patients seek psychological, educational or rehabilitation services when they are experiencing high levels of symptoms, and, on average, symptoms subside to some degree over the course of a trial, regardless of whether treatment is provided. For instance, in placebo groups in antidepressant trials and in usual care groups in depression psychotherapy trials, approximately $40 \%$ of patients with major depression remit. ${ }^{27}$ Since the course of SSc is highly unpredictable, and since symptom severity fluctuates substantially, tests of interventions that use non-RCT designs, including pre-post designs, would be difficult to interpret and could generate potentially misleading results. RCTs address this problem by randomly allocating participants to trial arms.

Eligible trials must be conducted with participants with SSc, verified by clinical diagnosis or based on participant self-report. If an RCT includes some participants with SSc, but other participants who do not have SSc, it will be included only if results for those with SSc are reported separately or if at least $80 \%$ of participants have SSc. 
Studies that report results from $<10$ participants per trial arm will be excluded.

Eligible interventions include, but are not limited to, physical or occupational therapy, rehabilitation, exercise, psychological, self-management, educational, diet or nutrition, nursing, podiatry, and oral or dental hygiene interventions. All pharmacological interventions, or interventions with a drug component, will be excluded. Interventions will be classified as having a drug component if any form of the active intervention ingredient was listed by the US Food and Drug Administration (FDA) in the Drugs@FDA database at the time of review. If not listed, investigators will consult other relevant sources to determine drug status. Use of probiotics will be included as a dietary or nutrition intervention if delivered as a food product similar to products that could be obtained outside of a medical intervention (eg, yoghurt). They will be excluded if they are products registered as a drug or delivered in pill format. Biologicals will be excluded, even if autologous (eg, skin grafting, stem cells), regardless of regulation status. Eligible intervention comparators will include: (1) any inactive control condition (eg, no treatment, waitlist control, usual care) or (2) another eligible intervention designed to improve health outcomes in SSc or to improve delivery of services. At least one measured outcome must pertain to physical or psychological health or to aspects of delivery of health or support services (eg, e-health, support groups).

\section{Search strategy}

We will include all eligible RCTs from a previous systematic review, ${ }^{13}$ which searched for trials published between 1990 and March 2014. Additional trials will be sought using a similar, augmented, search strategy from January 2014 to the present, developed and performed by a health sciences librarian and peer-reviewed ${ }^{28}$ (see online supplemental file 1). Articles for review will be identified from the MEDLINE, Embase, CINAHL, PsycINFO, Cochrane Library and Web of Science databases. Searches will not be restricted by language or publication status. The previous review ${ }^{13}$ did not include trials on interventions to improve the delivery of health services or support services to individuals with SSc; however, since health services interventions have changed considerably in recent years, we will only consider delivery of services trials that have been published from January 2014 onwards.

In addition to database searches, we will manually review references from other relevant reviews, search clinical trial registries and query authors of included RCTs about unpublished trials. After the initial search, automated searches will be set for monthly updates to facilitate continual review and update.

\section{Selection of eligible studies}

The results of the initial search and subsequent searches will be into the systematic review software DistillerSR (Evidence Partners, Ottawa, Canada) where duplicate references will be identified and removed. A two-stage process will be used to determine the eligibility of each publication, using a predefined inclusion and exclusion coding manual (see online supplemental file 2). Two independent investigators will review the titles and abstracts of the articles identified through the search strategy in random order. If either of the two reviewers deems an article to be potentially eligible for inclusion, a full-text review of the article will be completed independently by two reviewers. Any disagreements that may arise at the full-text level will be resolved by consensus, with a third reviewer consulted as necessary.

\section{Data extraction}

For each included RCT, one investigator will extract data using a prespecified standardised form in DistillerSR. A second reviewer will validate the extracted data using the DistillerSR Quality Control function. Reviewers will extract (1) publication characteristics (eg, first author last name, publication year, journal, funding source, author conflicts of interest); (2) population characteristics and demographics (eg, country, study eligibility criteria, recruitment method, number of participants, age, sex, type of SSc, disease duration, symptom thresholds); (3) intervention components (eg, descriptions of the intervention and comparator, number of participants randomised to intervention and control groups, number of participants analysed in both groups, intervention length, intervention setting (where delivered), number of sessions, length of sessions, qualifications of intervention providers, tailoring, modifications and fidelity adherence, as described by the Template for Intervention and Description and Replication Checklist ${ }^{29}$ and (4) health or service provision outcomes. See online supplemental file 3 for variables to be extracted. Disagreements will be resolved by consensus, with a third investigator consulted as necessary.

Two reviewers will independently assess included studies for risk of bias using the revised cochrane risk of bias tool for randomised trials. ${ }^{30}$ The tool includes five domains through which bias could be introduced; they are assessed and scored according to whether study characteristics reflect low, high or some concerns regarding risk of bias. Domains include (1) risk of bias due to the randomisation process; (2) deviations from the intended intervention; (3) missing outcome data; (4) outcome measurement and (5) selection of the reported result. Disagreements will be resolved by consensus, and a third investigator will be consulted as necessary.

\section{Data analysis}

Meta-analyses will be considered if $\geq 2$ eligible RCTs report similar non-pharmacological interventions and comparable health outcomes and if the trials are of sufficiently high general quality to draw conclusions, based on judgements about risk of bias, sample size and risk of publication bias. RCTs that are not meta-analysed will be described qualitatively. When studies are synthesised meta-analytically, data will be pooled using the 
DerSimonian Laird random effects model.$^{31}$ For dichotomous outcomes, we will report relative risks between groups with 95\% CIs. For continuous outcomes, Hedges' $\mathrm{g}$ will be used to calculate standardised mean differences for continuous outcomes. ${ }^{32}$ We will prioritise postintervention comparisons adjusted for baseline values, then unadjusted comparisons, followed by comparisons of change scores. If multiple measures are used to assess the same outcome in a study, effect sizes from all relevant measures will be synthesised within the study before being entered into the meta-analysis. If we are not able to use a study's data in a meta-analysis because, for instance, only $\mathrm{p}$ values are reported, we will begin by querying the authors for the relevant results. If the full results are not able to be obtained, we will present what was provided in publications in tables. The $\mathrm{I}^{2}$ statistic will be used to assess the heterogeneity of included trials. ${ }^{33}$ Publication bias will be examined using funnel plots if there are at least 10 studies included for a given intervention.

\section{Patient and public involvement}

The research team includes people with SSc who are actively involved as members of the Scleroderma Patientcentered Intervention Network Patient (SPIN) Advisory Board. They have provided input on the protocol and study design and will be involved in review, interpretation and dissemination of results.

\section{ETHICS AND DISSEMINATION}

Ethics approval was not required for this systematic review because we will be including only published aggregate data.

To disseminate results, we will post initial and ongoing results via a website (https://www.spinsclero.com/en/ projects/non-pharm-interventions) and publish results periodically via peer-reviewed journal publications. Updated results will be updated to the website as soon as new trials are identified. We will submit updated results from journal publication when the author team identifies that there are substantive changes in the evidence base. We will further disseminate results to the international scleroderma patient community through the SPIN social media accounts and by distribution of announcements to SPIN's patient organisation partners. Systematic review team members present regularly at national and international scleroderma patient congresses, and we plan to disseminate results at those congresses.

\footnotetext{
Author affiliations

${ }^{1}$ Lady Davis Institute of the Jewish General Hospital, Montreal, Quebec, Canada

${ }^{2}$ Department of Psychology, McGill University, Montreal, Quebec, Canada

${ }^{3}$ Department of Psychiatry, McGill University, Montreal, Quebec, Canada

${ }^{4}$ Department of Clinical Psychology, Behavioural Science Institute, Radboud Universiteit, Nijmegen, The Netherlands

${ }^{5}$ Schulich Library of Physical Sciences, Life Sciences, and Engineering, McGill University, Montreal, Quebec, Canada

${ }^{6}$ Department of Neurobiology, Care Sciences and Society, Division of Physiotherapy, Karolinska Institutet, Stockholm, Sweden

${ }^{7}$ Faculty of Kinesiology, University of Calgary, Calgary, Alberta, Canada
}

${ }^{8}$ Department of Medicine, McGill University Faculty of Medicine, Montreal, Quebec, Canada

${ }^{9}$ Tufts University School of Dental Medicine, Boston, Massachusetts, USA

${ }^{10}$ Department of Physiotherapy, Sunderby Hospital, Lulea, Sweden

${ }^{11}$ Service de Médecine Interne, Centre de Référence Maladies Auto-immunes et Systémiques Rares d'lle de France, Hopital Cochin Assistance Publique - Hôpitaux de Paris (APHP), Paris, France

${ }^{12}$ APHP-CUP, Hôpital Cochin, Université de Paris, Paris, France

${ }^{13}$ School of Nursing, Duke University, Durham, North Carolina, USA

${ }^{14}$ Departments of Pediatrics and Internal Medicine and College of Population Health, University of New Mexico, Albuquerque, California, USA

${ }^{15}$ Scleroderma Societies of Canada and Ontario, Hamilton, Ontario, Canada

${ }^{16}$ FESCA Patient Research Partner, Utrecht, The Netherlands

${ }^{17}$ Sclérodermie Québec, Montreal, Quebec, Canada

${ }^{18}$ Departments of Medicine and of Epidemiology, Biostatistics \& Occupational Health, McGill University, Montreal, Quebec, Canada

${ }^{19}$ Research Institute of the McGill University Health Centre, Montreal, Quebec, Canada, Montreal, Quebec, Canada

${ }^{20}$ Respiratory Epidemiology and Clinical Research Unit, McGill University Health Centre, Montreal, Quebec, Canada

${ }^{21}$ Department of Educational and Counselling Psychology, McGill University,

Montreal, Quebec, Canada

${ }^{22}$ Biomedical Ethics Unit, McGill University, Montreal, Quebec, Canada

Contributors M-ND, AC-J, LK, RSH, AB and BDT contributed to the initial conceptual framework and design of the systematic review. AK, CB, NC-R, MH, DML, MM, LM, RW, EYJ, MS, JW and GG reviewed the initial framework and provided input. JB developed the database search strategy and AK carried out searches. M-ND, AC-J, LK, RSH and BDT drafted this protocol. All authors provided critical revisions of the protocol and approved the submission of the final manuscript. BDT is the guarantor.

Funding AC-J was supported by a McGill University Faculty of Medicine Solvay Fellowship and by a McGill University Delta Upsilon Scholarship. RSH was supported by a Mitacs postdoctoral fellowship, supported by Scleroderma Canada. BDT was supported by a Tier 1 Canada Research Chair.

Competing interests None declared.

Patient consent for publication Not required.

Provenance and peer review Not commissioned; externally peer reviewed.

Supplemental material This content has been supplied by the author(s). It has not been vetted by BMJ Publishing Group Limited (BMJ) and may not have been peer-reviewed. Any opinions or recommendations discussed are solely those of the author(s) and are not endorsed by BMJ. BMJ disclaims all liability and responsibility arising from any reliance placed on the content. Where the content includes any translated material, BMJ does not warrant the accuracy and reliability of the translations (including but not limited to local regulations, clinical guidelines, terminology, drug names and drug dosages), and is not responsible for any error and/or omissions arising from translation and adaptation or otherwise.

Open access This is an open access article distributed in accordance with the Creative Commons Attribution Non Commercial (CC BY-NC 4.0) license, which permits others to distribute, remix, adapt, build upon this work non-commercially, and license their derivative works on different terms, provided the original work is properly cited, appropriate credit is given, any changes made indicated, and the use is non-commercial. See: http://creativecommons.org/licenses/by-nc/4.0/.

ORCID iD

Brett D Thombs http://orcid.org/0000-0002-5644-8432

\section{REFERENCES}

1 Mayes MD. Systemic sclerosis: clinical features. In: Klippel JH, Stone $\mathrm{JH}$, White $\mathrm{PH}$, eds. Primer on the rheumatic diseases. New York, NY: Springer Science \& Business Media, 2008: 343-50.

2 Gelber AC, Manno RL, Shah AA, et al. Race and association with disease manifestations and mortality in scleroderma: a 20-year experience at the Johns Hopkins scleroderma center and review of the literature. Medicine 2013;92:191-205.

3 Kwakkenbos L, Delisle VC, Fox RS, et al. Psychosocial aspects of scleroderma. Rheum Dis Clin North Am 2015;41:519-28. 
4 Haythornthwaite JA, Heinberg LJ, McGuire L. Psychologic factors in scleroderma. Rheum Dis Clin North Am 2003;29:427-39.

5 Bassel M, Hudson M, Taillefer SS, et al. Frequency and impact of symptoms experienced by patients with systemic sclerosis: results from a Canadian national survey. Rheumatology 2011;50:762-7.

6 Thombs BD, Jewett LR, Kwakkenbos L, et al. Major depression diagnoses among patients with systemic sclerosis: baseline and onemonth followup. Arthritis Care Res 2015;67:411-6.

7 Razykov I, Levis B, Hudson M, et al. Prevalence and clinical correlates of pruritus in patients with systemic sclerosis: an updated analysis of 959 patients. Rheumatology 2013;52:2056-61.

8 Milette K, Hudson M, Körner A, et al. Sleep disturbances in systemic sclerosis: evidence for the role of gastrointestinal symptoms, pain and pruritus. Rheumatology 2013;52:1715-20.

9 Jewett LR, Kwakkenbos L, Thombs BD. Psychosocial issues and care for patients with systemic sclerosis. In: Varga J, Denton $\mathrm{CP}$, Wigley FM, et al, eds. Scleroderma: from pathogenesis to comprehensive management. Cham: Springer International Publishing, 2017: 2. 615-21.

10 Hudson M, Thombs BD, Steele R, et al. Health-Related quality of life in systemic sclerosis: a systematic review. Arthritis Rheum 2009;61:1112-20.

11 Thombs BD, Taillefer SS, Hudson M, et al. Depression in patients with systemic sclerosis: a systematic review of the evidence. Arthritis Rheum 2007; 57:1089-97.

12 Spierings J, van den Ende C, Schriemer R, et al. Optimal care for systemic sclerosis patients: recommendations from a patientcentered and multidisciplinary mixed-method study and working conference. Clin Rheumatol 2019;38:1007-15.

13 Willems LM, Vriezekolk JE, Schouffoer AA, et al. Effectiveness of nonpharmacologic interventions in systemic sclerosis: a systematic review. Arthritis Care Res 2015;67:1426-39.

14 Liem SIE, Vliet Vlieland TPM, Schoones JW, et al. The effect and safety of exercise therapy in patients with systemic sclerosis: a systematic review. Rheumatol Adv Pract 2019;3:rkz044.

15 Smith E, Pauling JD. The efficacy of dietary intervention on gastrointestinal involvement in systemic sclerosis: a systematic literature review. Semin Arthritis Rheum 2019;49:112-8.

16 Rannou F, Boutron I, Mouthon L, et al. Personalized physical therapy versus usual care for patients with systemic sclerosis: a randomized controlled trial. Arthritis Care Res 2017;69:1050-9.

17 Khanna D, Serrano J, Berrocal VJ. Randomized controlled trial to evaluate an internet-based self-management program in systemic sclerosis. Arthritis Care Res 2019;71:435-47.

18 Filippetti M, Cazzoletti L, Zamboni F, et al. Effect of a tailored home-based exercise program in patients with systemic sclerosis: a randomized controlled trial. Scand J Med Sci Sports 2020;30:1675-84.

19 Uras C, Mastroeni S, Tabolli S, et al. A comparison between two educational methods in the rehabilitation of the microstomia in systemic sclerosis: a randomized controlled trial. Clin Rehabil 2019;33:1747-56.

20 The scleroderma patient-centered intervention network hand program (SPIN-HAND). NCT03419208. Available: https://clinicaltrials. gov/ct2/show/NCT03419208?term=SPIN-HAND\&draw=2\&rank=1 [Accessed March 30, 2020].

21 Thombs BD, Aguila K, Dyas L, et al. Protocol for a partially nested randomized controlled trial to evaluate the effectiveness of the scleroderma patient-centered intervention network support group leader education (SPIN-SSLED) program. Trials 2019;20:717.

22 Thombs BD, Kwakkenbos L, Carrier M-E, et al. Protocol for a partially nested randomised controlled trial to evaluate the effectiveness of the scleroderma patient-centered intervention network COVID-19 home-isolation activities together (SPIN-CHAT) program to reduce anxiety among at-risk scleroderma patients. J Psychosom Res 2020;135:110132.

23 Higgins JPT, Thomas J, Chandler J. Cochrane Handbook for systematic reviews of interventions version 6.1 (updated September 2020). Cochrane, 2020. Available: www.training.cochrane.org/ handbook [Accessed March 30, 2021].

24 Brooker J, Synnot A, McDonald S. Guidance for the production and publication of Cochrane living systematic reviews: cochrane reviews in living mode (version December 2019). Cochrane, 2019. Available: https://community.cochrane.org/review-production/ production-resources/living-systematic-reviews [Accessed March 30, 2021].

25 Moher D, Shamseer L, Clarke M, et al. Preferred reporting items for systematic review and meta-analysis protocols (PRISMA-P) 2015 statement. Syst Rev 2015;4:1.

26 Liberati A, Altman DG, Tetzlaff J, et al. The PRISMA statement for reporting systematic reviews and meta-analyses of studies that evaluate health care interventions: explanation and elaboration. $J$ Clin Epidemiol 2009;62:e1-34

27 Cuijpers $\mathrm{P}$. The challenges of improving treatments for depression. JAMA 2018;320:2529-30.

28 McGowan J, Sampson M, Salzwedel DM, et al. PRESS Peer Review of Electronic Search Strategies: 2015 Guideline Statement. J Clin Epidemiol 2016;75:40-6.

29 Hoffmann TC, Glasziou PP, Boutron I, et al. Better reporting of interventions: template for intervention description and replication (TIDieR) checklist and guide. BMJ 2014;348:g1687.

30 Sterne JAC, Savović J, Page MJ, et al. Rob 2: a revised tool for assessing risk of bias in randomised trials. BMJ 2019;366:14898.

31 DerSimonian R, Laird N. Meta-Analysis in clinical trials. Control Clin Trials 1986;7:177-88.

32 Hedges LV. Estimation of effect size from a series of independent experiments. Psychol Bull 1982;92:490-9.

33 Higgins JPT, Thompson SG. Quantifying heterogeneity in a metaanalysis. Stat Med 2002;21:1539-58. 